\title{
Terahertz and Far-infrared Spectroscopic Estimation of Vinyl Acetate Content in Ethylene-Vinyl Acetate Copolymer
}

\author{
Tomoyuki Izutsu ${ }^{1}$, Daisuke Odaka ${ }^{1}$, Marina Komatsu ${ }^{1}$, \\ and Yoshimichi Ohki ${ }^{1,2}$ \\ ${ }^{1}$ Department of Electrical Engineering and Bioscience \\ ${ }^{2}$ Research Institute for Materials Science and Technology \\ Waseda University \\ Shinjuku-ku, Tokyo 169-8555, Japan \\ t.izutsu1016@ruri.waseda.jp
}

\author{
Maya Mizuno \\ Applied Electromagnetic Research Institute \\ National Institute of Information and Communications \\ Technology \\ Koganei-shi, Tokyo 184-8795, Japan
}

\author{
Yoshiaki Nakamura and Naofumi Chiwata \\ Cable Materials Company \\ Hitachi Metals \\ Hitachi-shi, Ibaraki 319-1414, Japan
}

\begin{abstract}
For the purpose of estimating the content of vinyl acetate (VA) in ethylene-vinyl acetate copolymer (EVA), optical absorption spectra were measured in a frequency range from 0.5 to 21.0 THz for sheets of EVA containing different contents of VA from 12 to $60 \%$ by weight and a sheet of low-density polyethylene containing no VA. As a result, it has become clear that EVA exhibits relatively sharp absorption at 2.5, 6.7, and 10.5 $\mathrm{THz}$ and rather broad absorption at 11 to $17 \mathrm{THz}$ and 17 to 20 THz. All these absorption peaks and bands are in proportion to the content of VA, up to certain frequency-dependent contents. Using these proportionalities, the content of VA in EVA can be estimated quantitatively. Especially, the absorption at $2.5 \mathrm{THz}$ shows a good accuracy similar to the method recommended by an international standard.
\end{abstract}

Keywords-terahertz wave; terahertz time domain spectroscopy; far-infrared spectroscopy; ethylene-vinyl acetate copolymer; vinyl acetate

\section{INTRODUCTION}

Ethylene-vinyl acetate copolymer (EVA) is industrially valuable, since it is used for cable insulation as well as for films in various fields [1]. As the content of vinyl acetate (VA) in EVA increases, EVA becomes soft and polar and its permittivity increases [1]. Namely, the estimation of the content of VA in EVA is industrially important for determining its mechanical and electrical properties. At present, several methods including Fourier-transform infrared (FT-IR) spectroscopy are recommended in several standards such as ISO 8985 and Japanese Industrial Standard JIS K 7192 [2]. Especially, for manufacturing EVA, a simpler and more reliable method is desirable.

This work was partly supported by a Grant-in-Aid from the Japan Society for the Promotion of Science (JSPS) for JSPS Fellows $(25 \cdot 2605)$.
From this viewpoint, the authors focused on terahertz (THz) and FT far-infrared (FIR) absorption spectroscopy. Here, the $\mathrm{THz}$ waves are the electromagnetic waves in a frequency range from 0.1 to $10 \mathrm{THz}$ with wave numbers from 3.3 to $333 \mathrm{~cm}^{-1}$. It has been known that $\mathrm{THz}$ absorption spectroscopy can detect various intermolecular vibrations, lattice vibrations, and skeletal vibrations [3-8]. Therefore, it is expected that we can obtain valuable information on various properties of a substance if we use $\mathrm{THz}$ spectroscopy in addition to conventional instrumental analyses such as X-ray diffraction and FT-IR. This work was carried out to examine the possibility whether $\mathrm{THz}$ and FT-FIR absorption spectroscopy can be used as a tool to estimate the VA content in EVA. Furthermore, the reliability was compared between the THz-FIR method and the FT-IR method.

\section{EXPERIMENTAL}

Sheets of EVA, about $1.0 \mathrm{~mm}$ thick, containing different contents of VA from 12 to $60 \%$ by weight and a sheet of lowdensity polyethylene (LDPE), containing no VA, with a similar thickness were prepared. For these sheet samples, $\mathrm{THz}$ absorption spectra were obtained in a frequency range from 0.5 to 4.0 THz by a THz time domain spectrometer (TDS, TochigiNikon, Rayfact SpecTera RS-01020) in vacuum at room temperature. Furthermore, FT-FIR absorption spectra were obtained in a frequency range from 3.0 to $21.0 \mathrm{THz}$ by a spectrometer (JASCO, VIR-F) in the atmospheric air at room temperature. Moreover, FT-IR spectra were measured in a wave number range from 3600 to $2400 \mathrm{~cm}^{-1}$ using a spectrometer (JASCO, FT/IR-4200) in the atmospheric air at room temperature. 


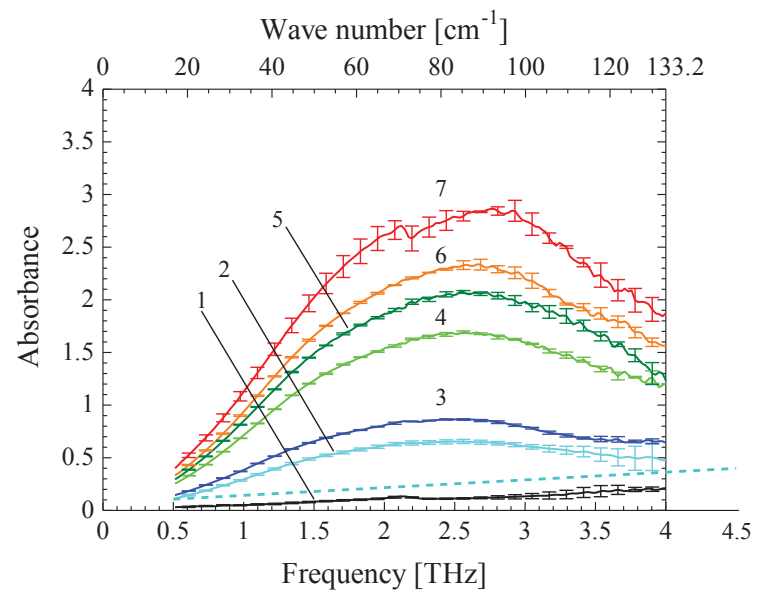

Fig. 1. Absorption spectra of LDPE (1, black) and EVA with various VA contents at frequencies from 0.5 to $4.0 \mathrm{THz} ; 2$, light blue: VA contents of $12 \%, 3$, dark blue: $17 \%$, 4, light green: $28 \%, 5$, dark green: $33 \%, 6$, orange: $42 \%$, and 7 , red: $60 \%$. Each spectrum was drawn as the average of three measurements and each vertical bar represents the \pm one standard deviation of the measurement values. The light blue dashed straight line, which connects two absorbance values at 0.5 and $4.5 \mathrm{THz}$ (see Fig. 2), was drawn to remove the background noise from the spectrum of the $12 \%$ sample.

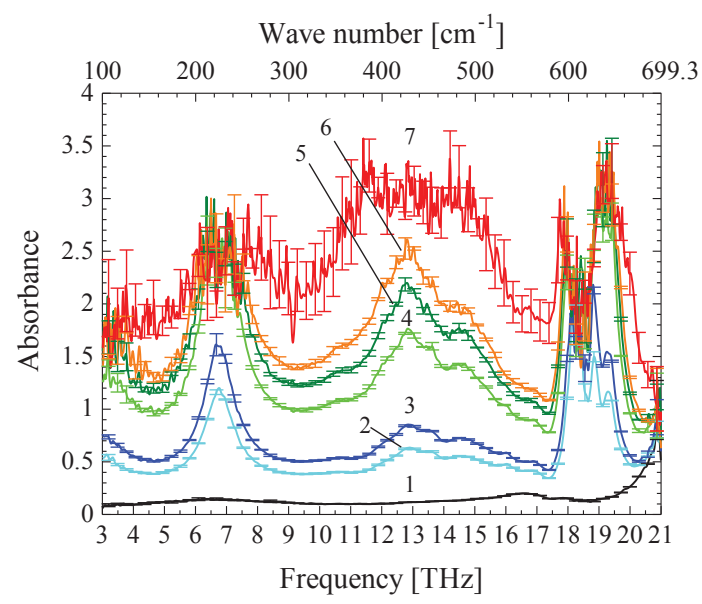

Fig. 2. Absorption spectra of LDPE (1, black) and EVA with various VA contents at frequencies from 3.0 to $21.0 \mathrm{THz}$; 2 , light blue: VA contents of $12 \%, 3$, dark blue: $17 \%$, 4, light green: $28 \%, 5$, dark green: $33 \%, 6$, orange: $42 \%$, and 7 , red: $60 \%$. Each spectrum was drawn as the average of three measurements and each vertical bar represents the \pm one standard deviation of the measurement values.

\section{RESULTS AND DISCUSSION}

\section{A. THz and Far-infrared Absorption Spectra}

Fig. 1 shows THz absorption spectra of EVA and LDPE in a frequency range from 0.5 to $4.0 \mathrm{THz}$. Each spectrum was drawn as the average of three measurements and each vertical bar represents the \pm one standard deviation of the measurement values. First, LDPE shows an absorption peak at $2.15 \mathrm{THz}$. This absorption is attributable to a crystalline region in LDPE [9]. Therefore, there should be no direct relation between its
TABle I. Peak Frequencies and the Full Widths at HalF MAXIMA OF EIGHT ABSORPTION COMPONENTS ASSUMED FOR SEPARATING THE ABSORPTION SPECTRA WITH LORENTZIAN CURVES

\begin{tabular}{|c|c|}
\hline Peak Frequency [THz] & Full Width at Half Maximum [THz] \\
\hline 6.76 & 0.96 \\
\hline 10.5 & 0.99 \\
\hline 12.8 & 1.50 \\
\hline 13.5 & 0.48 \\
\hline 14.5 & 1.32 \\
\hline 18.2 & 0.45 \\
\hline 18.9 & 0.30 \\
\hline 19.3 & 0.51 \\
\hline
\end{tabular}

intensity and the VA content in EVA. On the other hand, EVA sheets have a broad but strong absorption peak, which shows its maximum intensity at around $2.5 \mathrm{THz}$, in addition to the weak absorption at $2.15 \mathrm{THz}$. The intensity of this broad absorption increases with an increase in content of VA. The assignment of this broad absorption at around $2.5 \mathrm{THz}$ was already reported as an intermolecular oscillation activated by the large dipole moment of the acetate group [10].

Fig. 2 shows FIR absorption spectra of EVA and LDPE in a frequency range from 3.0 to $21.0 \mathrm{THz}$. While LDPE shows no meaningful absorption peaks, EVA sheets have a sharp absorption peak at $6.7 \mathrm{THz}$ and a broad absorption band with seemingly multiple peaks stretching from 11 to $17 \mathrm{THz}$ as well as a rather sharp and strong absorption band with multiple peaks at 17 to $20 \mathrm{THz}$. Furthermore, the EVA sheet with a relatively high content of VA shows a weak absorption peak at $10.5 \mathrm{THz}$. Among the above-mentioned four peaks and bands, the absorption peak at $6.7 \mathrm{THz}$ was assigned tentatively to internal mode of the acetate group [11].

\section{B. Dependence of THz and Far-infrared Absorption Spectra on the VA Content}

The absorption spectra shown in Fig. 2 overlap each other. In order to obtain the intensity of each absorption, background noises should be removed. First, the effective absorption coefficient $(\alpha)$ was calculated for each sample in the form of the spectrum as a function of frequency by dividing its absorbance by its thickness. Secondly, the $\alpha$ spectrum of the LDPE sheet was subtracted from the $\alpha$ spectrum of the EVA sheets in a frequency range from 3.0 to $21.0 \mathrm{THz}$. Here, the spectrum obtained by the subtraction is called the $\Delta \alpha$ spectrum. Thirdly, two straight lines were drawn on the $\Delta \alpha$ spectrum by connecting them from the minimum point of $\Delta \alpha$ at around 17 to $18 \mathrm{THz}$ to two minimum points of $\Delta \alpha$ at around 4 to $5 \mathrm{THz}$ and at around 20 to $21 \mathrm{THz}$. By subtracting absorption spectra indicated with the two straight lines from the $\Delta \alpha$ spectra, $\Delta \Delta \alpha$ spectra were obtained. Fourthly, peaks appearing in the $\Delta \Delta \alpha$ spectra were separated by assuming eight Lorentzian curves. For the peak separation, the frequency and the full width at half maximum of each peak were fixed at the values shown in Table I. Fifthly, the area of each peak or integrated absorption intensity, which has a unit of $\mathrm{cm}^{-2}$, was calculated as the integral over the wave number. 


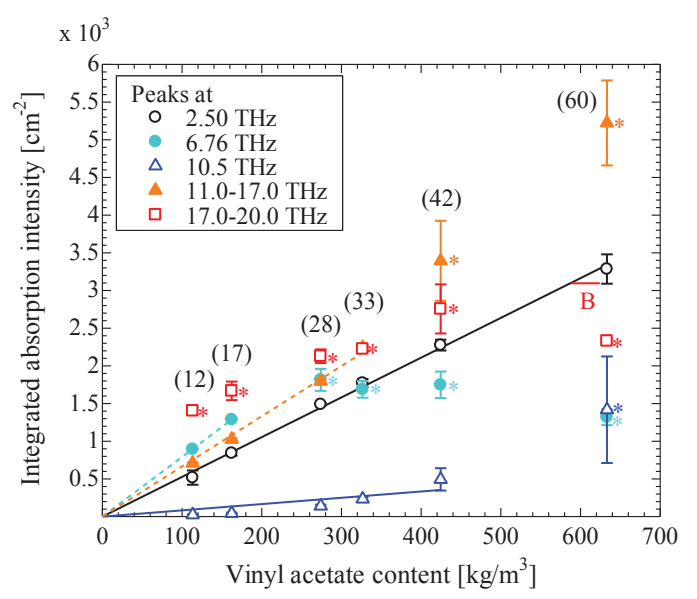

Fig. 3. Integrated intensity as a function of the content of VA for each absorption. The data are averages of three measurements and vertical bars represent their \pm one standard deviations. The asterisk mark * indicates that the absorption is either composed of several sub-peaks or saturated. The red horizontal line labeled B was drawn for the purpose of the estimation of the measurement errors. The figures in the parentheses denote the VA contents in \% by weight.

As for Fig. 1, first, as in the case of Fig. 2, $\Delta \alpha$ spectra were obtained by subtracting the $\alpha$ spectrum of the LDPE sheet from the $\alpha$ spectra of the EVA sheets. Secondly, a straight line was drawn for each $\Delta \alpha$ by connecting it from the value at $0.5 \mathrm{THz}$ to the minimum value of $\Delta \alpha$ appearing in Fig. 2 in a frequency range from 4 to $5 \mathrm{THz}$. Note that the light blue dashed line in Fig. 1 is an example of the straight line drawn for EVA with VA content of $12 \%$. Thirdly, $\Delta \Delta \alpha$ was calculated by subtracting the absorption spectrum indicated with the straight line from each $\Delta \alpha$. Fourthly, the integrated absorption intensity of the $2.5-\mathrm{THz}$ peak was obtained.

Fig. 3 shows the integrated absorption intensity of each $\Delta \Delta \alpha$. Here, the unit of VA content on the abscissa was converted from $\%$ by weight to $\mathrm{kg} / \mathrm{m}^{3}$ by assuming the densities of LDPE and polyvinyl acetate as 0.92 and 1.17 $\mathrm{g} / \mathrm{cm}^{3}[12,13]$, respectively. According to the conversion, the VA contents of $12,17,28,33,42$, and $60 \%$ by weight correspond to $113,162,274,327,424$, and $633 \mathrm{~kg} / \mathrm{m}^{3}$, respectively. Here, as shown in Fig. 2, several absorption peaks seem to overlap each other at frequencies from 11 to $17 \mathrm{THz}$ and from 17 to $20 \mathrm{THz}$. If some of these peaks are estimated too much, the others should be underestimated, which makes the estimation of peak intensity difficult. However, the purpose of this work is not to determine the intensity of every component peak but to estimate the VA content. Accordingly, as for the two multi-peak absorption bands, the total integrated absorption intensity was shown in Fig. 3 without trying peak separation. Furthermore, it appears that several absorption peaks or bands tend to split into sub-peaks or become saturated at relatively high contents of VA, probably due to low signalto-noise ratio. In such a case, an asterisk mark $(*)$ was put in Fig. 3 on the corresponding integrated intensity.

Based on the above, except for the absorption band at around 17 to $20 \mathrm{THz}$, which seems saturated even at $12 \%$, the integrated absorption intensities at frequencies of 2.50, 6.76,

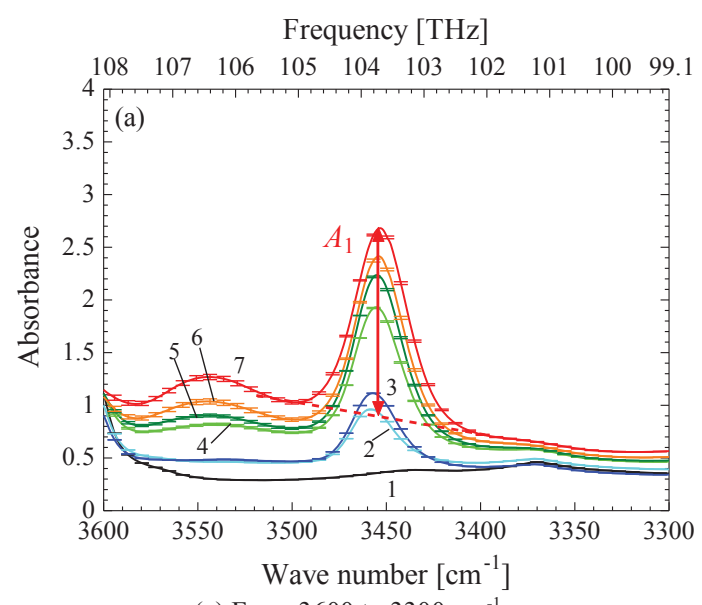

(a) From 3600 to $3300 \mathrm{~cm}^{-1}$.

Frequency [THz]

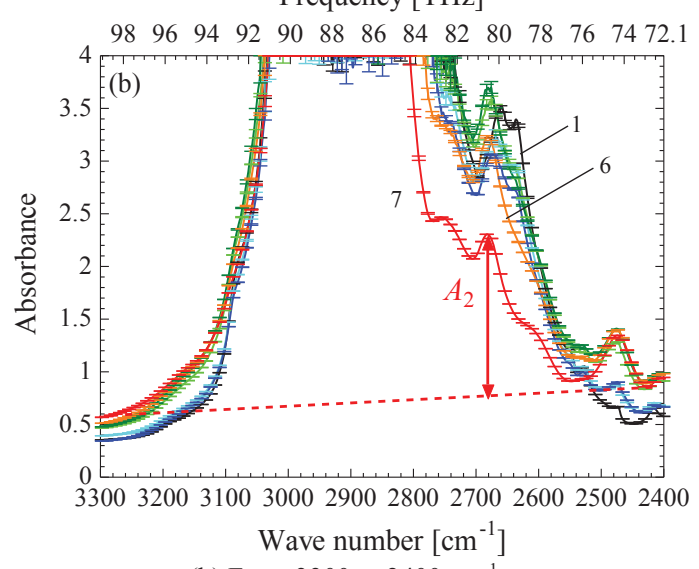

(b) From 3300 to $2400 \mathrm{~cm}^{-1}$.

Fig. 4. Absorption spectra of LDPE (1, black) and EVA with various VA contents at wave numbers from 3600 to $3300 \mathrm{~cm}^{-1}$ (a) and from 3300 to $2400 \mathrm{~cm}^{-1}$ (b); 2, light blue: VA contents of $12 \%$, 3, dark blue: $17 \%$, 4, light green: $28 \%$, 5, dark green: $33 \%$, 6 , orange: $42 \%$, and 7 , red: $60 \%$. Each spectrum was drawn as the average of three measurements and each vertical bar represents the \pm one standard deviation of the measurement values. The red dashed vertical line, which connects two absorbance values at 3520 and $3400 \mathrm{~cm}^{-1}$ or those at 3280 and $2430 \mathrm{~cm}^{-1}$, was drawn to obtain the absorbance $A_{1}$ or $A_{2}$ of the sample with the VA content of $60 \%$.

and 10.5, and the intensity in a range from 11.0 to $17.0 \mathrm{THz}$ show a good proportionality up to the VA content of $60,17,42$, and $33 \%$, respectively. Therefore, from the slopes of the straight lines shown in Fig. 3, the integrated absorption intensity per unit amount or per $1.0 \mathrm{~kg} / \mathrm{m}^{3}$ of VA can be calculated to be around $5.3,7.9,0.83$, and $6.7 \mathrm{~m}^{3} /\left(\mathrm{kg} \cdot \mathrm{cm}^{2}\right)$ for the absorption at $2.50,6.76,10.5$, and 11.0 to $17.0 \mathrm{THz}$, respectively.

\section{IR Absorption Spectra}

Fig. 4 shows IR absorption spectra of EVA and LDPE in wave number ranges from 3600 to $3300 \mathrm{~cm}^{-1}$ (a) and from 3300 to $2400 \mathrm{~cm}^{-1}$ (b). The VA content can be estimated from these absorption spectra by a method specified by JIS [2]. The two red dashed straight lines were drawn for understanding the 


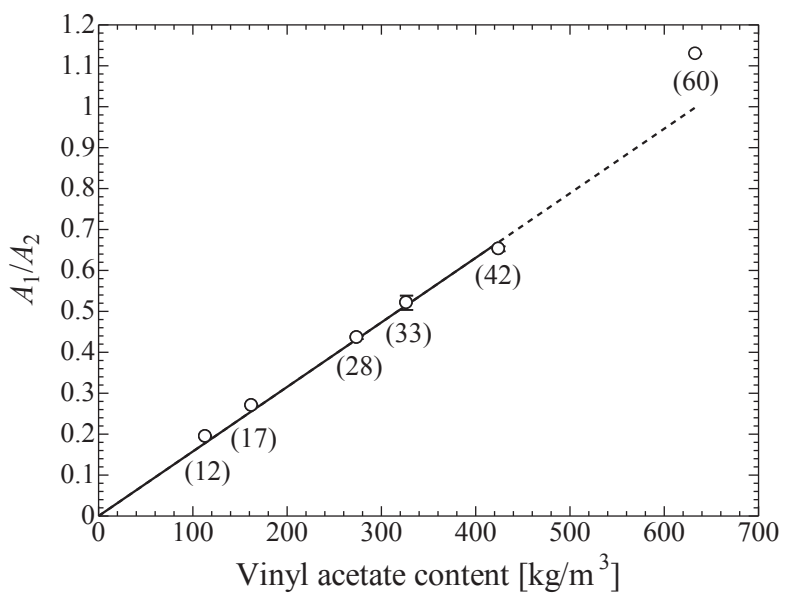

Fig. 5. Absorption ratio of $A_{1}$ (at $3460 \mathrm{~cm}^{-1}$ ) to $A_{2}$ (at $2678 \mathrm{~cm}^{-1}$ ) as a function of the content of VA. The data are averages of three measurements and vertical bars represent their \pm one standard deviations. The figures in the parentheses denote the VA contents in $\%$ by weight.

method, taking an example for EVA with VA content of $60 \%$. In Fig. 4 (a), EVA has an absorption peak at around $3460 \mathrm{~cm}^{-1}$ due to $\mathrm{C}=\mathrm{O}$ bonds. First, the absorption intensity at around $3460 \mathrm{~cm}^{-1}\left(A_{1}\right)$ shown in Fig. 4 (a) should be measured as the peak height over the straight line connecting the values at 3520 and $3400 \mathrm{~cm}^{-1}$. Next, as for Fig. 4 (b), the absorption intensity due to $\mathrm{CH}_{2}$ bonds at around $2678 \mathrm{~cm}^{-1}\left(A_{2}\right)$ should be measured similarly as the height over the straight line shown in Fig. 4 (b) connecting the values at 3280 and $2430 \mathrm{~cm}^{-1}$. The VA content can be known by the ratio $A_{1} / A_{2}$ [2].

In Fig. 5, black open circles show the ratios $A_{1} / A_{2}$, while the black solid line shows the approximation up to the VA content of $42 \%$. The ratio at the VA content of $60 \%\left(=633 \mathrm{~kg} / \mathrm{m}^{3}\right)$ deviates upward from the extrapolation of the solid line. Similar upward deviation from the linear relation is also mentioned in JIS K 7192, if the VA content exceeds 40 or $50 \%[2]$.

\section{Accuracy of the Estimation of VA Content}

Each data point shown in Figs. 3 and 5 should have an error. The red horizontal line denoted by the letter $B$ in Fig. 3 connects the lower limit of the error bar put on the black open circle at $633 \mathrm{~kg} / \mathrm{m}^{3}$ and the straight line drawn to show the corresponding integrated absorption intensity per unit VA content. The length of the line B corresponds to $47 \mathrm{~kg} / \mathrm{m}^{3}$, which is $7.4 \%$ of the VA content of $633 \mathrm{~kg} / \mathrm{m}^{3}$. That is to say, the estimation error by this definition is $7.4 \%$ at this VA content. Similar estimation errors were calculated for all the upper and lower limits of the error bars for the $2.5-\mathrm{THz}$ integrated absorption intensity, which has a good proportionality up to the VA content of $60 \%$. As a result, the average of the estimation errors was calculated to be $5.9 \%$. Estimation errors were calculated similarly for the data shown in Fig. 5 except for the value at the VA content of $60 \%$, and their average was found to be $4.2 \%$. Therefore, if we compare the two absorption spectra in the range stretching over the $\mathrm{THz}$ and FIR frequencies and in the IR range, the $2.5-\mathrm{THz}$ absorption exhibits similar accuracy as the IR method specified in ISO and JIS, while both the two ranges can estimate the VA content in EVA.

\section{CONCLUSIONS}

The possibility whether $\mathrm{THz}$ or FIR absorption spectroscopy can be used as a tool to estimate the VA content in EVA was examined. Absorption spectra were obtained for sheets of EVA containing different contents of VA from 12 to $60 \%$ by weight and a sheet of LDPE containing no VA at frequencies from 0.5 to $21.0 \mathrm{THz}$. As a result, EVA has absorption peaks at 2.5, 6.7, and $10.5 \mathrm{THz}$ and broad bands stretching from 11 to $17 \mathrm{THz}$ and from 17 to $20 \mathrm{THz}$. The integrated absorption intensity of each absorption peak or band increases with an increase in content of VA. Therefore, the content of VA in EVA can be estimated quantitatively by $\mathrm{THz}$ and FIR absorption spectroscopy. Especially, the 2.5-THz absorption exhibits a similar accuracy as the method recommended by an international or a domestic standard using FT-IR.

\section{REFERENCES}

[1] A. M. Henderson, "Ethylene-vinyl acetate (EVA) copolymers: A general review," IEEE Electr. Insul. Mag., vol. 9, pp. 30-38, 1993.

[2] "Plastics-Ethylene/vinyl acetate copolymer (EVAC) thermoplastics Determination of vinyl acetate content," JIS K 7192, 1999.

[3] S. Wietzke, C. Jansen, M. Reuter, T. Jung, D. Kraft, S. Chatterjee, B. M. Fischer, and M. Koch, "Terahertz spectroscopy on polymers: A review of morphological studies," J. Mol. Struct., vol. 1006, pp. 41-51, 2011.

[4] H. Hoshina, Y. Morisawa, H. Sato, H. Minamide, I. Noda, Y. Ozaki, and C. Otani, "Polarization and temperature dependent spectra of poly(3hydroxyalkanoate)s measured at terahertz frequencies," Phys. Chem. Chem. Phys., vol. 13, pp. 9173-9179, 2011.

[5] M. Komatsu, M. Hosobuchi, X. Xie, Y. Cheng, Y. Furukawa, M. Mizuno, K. Fukunaga, and Y. Ohki, "Terahertz absorption spectra of oxidized polyethylene and their analysis by quantum chemical calculations,” Jpn. J. Appl. Phys., vol. 53, pp. 092402 1-9, 2014.

[6] Y. Ohki, M. Okada, N. Fuse, K. Iwai, M. Mizuno, and K. Fukunaga, "Terahertz time-domain spectroscopic analysis of molecular behavior in polyamide nanocomposites," Appl. Phys. Express, vol. 1, pp. 122401 13, 2008.

[7] N. Fuse, T. Takahashi, Y. Ohki, R. Sato, M. Mizuno, and K. Fukunaga, "Terahertz spectroscopy as a new tool for insulating material analysis and condition monitoring," IEEE Electr. Insul. Mag., vol. 27, pp. 26-35, 2011.

[8] N. Fuse, R. Sato, M. Mizuno, K. Fukunaga, K. Itoh, and Y. Ohki, "Observation and analysis of molecular vibration modes in polylactide at terahertz frequencies," Jpn. J. Appl. Phys., vol. 49, pp. 102402 1-8, 2010.

[9] S. Krimm and M. I. Bank, "Assignment of the $71-\mathrm{cm}^{-1}$ band in polyethylene," J. Chem. Phys., vol. 42, 4059-4060, 1965.

[10] G. W. Chantry, J. W. Fleming, E. A. Nicol, H. A. Willis, and M. E. A. Cudby, "Liquid-lattice type absorption in ethylene-vinyl acetate copolymers," Infrared Physics, vol. 12, pp. 101-107, 1972.

[11] G. W. Chantry, J. W. Fleming, R. J. Cook, H. A. Willis, and M. E. A. Cudby, "Far infrared and microwave absorption in ethylene-vinyl acetate copolymers," Infrared Physics, vol. 13, pp. 157-160, 1973.

[12] J. Brandrup and E. H. Immergut, eds., "Polymer Handbook," WileyInterscience, p. V/17, 1989.

[13] Ibid., p. V/71. 